\title{
Utilization of Artificial Intelligence for Diagnosis and Management of Urinary Incontinence in Women Residing in Areas with Low Resources: An Overview
}

\author{
Amad Qureshi ${ }^{1 *}$, Aanchal Mathur ${ }^{1 *}$, Jonia Alshiek ${ }^{1,2,3}$, S. Abbas Shobeiri1,2, Qi Wei1" \\ ${ }^{1}$ George Mason University, Fairfax, USA \\ ${ }^{2}$ Inova Fairfax Hospital, Fairfax, USA \\ ${ }^{3}$ Technion Faculty of Medicine, Hillel Yaffe Medical Center, Hadera, Israel

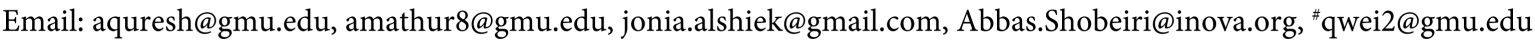

How to cite this paper: Qureshi, A., Mathur, A., Alshiek, J., Abbas Shobeiri, S. and Wei, Q. (2021) Utilization of Artificial Intelligence for Diagnosis and Management of Urinary Incontinence in Women Residing in Areas with Low Resources: An Overview. Open Journal of Obstetrics and $G y$ necology, 11, 403-418.

https://doi.org/10.4236/ojog.2021.114040

Received: February 26, 2021

Accepted: April 22, 2021

Published: April 25, 2021

Copyright $\odot 2021$ by author(s) and Scientific Research Publishing Inc. This work is licensed under the Creative Commons Attribution International License (CC BY 4.0).

http://creativecommons.org/licenses/by/4.0/ (c) (i) Open Access

\begin{abstract}
Urinary incontinence (UI) is a distressing condition involving involuntary loss of urine from the body. Urinary incontinence can negatively impact a person's overall quality of life and lead them into stages of embarrassment and depression. It is an underrepresented and undertreated condition prevalent in women, especially in low socioeconomic regions where women may not be able to express their concerns due to unawareness of diagnosis and treatment/management options. There are different diagnostic and management protocols for UI; however, utilizing artificially intelligent systems is not standard care. This paper overviews the use of artificial intelligence in women's health and as a means of cost-effectively diagnosing patients, and as an avenue for providing low-cost treatments to women that suffer from urinary incontinence in low-resource communities. Studies found that these systems, mainly utilizing artificial neural networks (ANNs) and convolutional neural networks (CNNs), served to be an effective method in diagnosing patients and providing an avenue for personalized treatment for improved patient outcomes. A simple artificial intelligence (AI) model utilizing Multilayer Perceptron (MLP) Networks was proposed to diagnose and manage urinary incontinence.
\end{abstract}

\section{Keywords}

Urinary Incontinence, Artificial Intelligence, Women's Health, Underdeveloped Regions 


\section{Introduction}

Urinary Incontinence (UI) is a distressing condition involving involuntary loss of urine from the body. It is estimated to affect over 423 million people worldwide, with a higher female population frequency [1]. Pregnancy, childbirth, menopause, or even injury can allow dysfunction of the bladder or the pelvic floor muscles and connective tissue, which would enable urine leakage to occur [2]. There are two common types of urinary incontinence-stress urinary incontinence and urgency urinary incontinence. Stress UI is the leakage of urine based on mechanical responses such as sneezing or jumping, which puts extra pressure on the pelvic floor and bladder [3]. Urgency UI or overactive bladder (OAB) is the leakage of urine due to the involuntary contraction of bladder muscles where the person would urinate under a sudden urge [4]. Although urinary incontinence is not lifethreatening, it is a condition that negatively impacts a person's overall quality of life, especially with women, as they may fall into a state of embarrassment and depression. The patients are often unable to express their concerns with their families and healthcare providers due to shame and the humiliation, and a lack of understanding of why the condition occurs. Women that suffer from this condition are often unaware of the diagnosis and management options, so UI is considered to be one of the more commonly underdiagnosed and underrepresented medical conditions globally [5].

\section{Focus of the Research}

Currently, some studies examine the general diagnostic and management protocol for urinary incontinence, including exercise, medication, and devices. Still, a limited number of these studies give an overview of artificial intelligence as a means of diagnosis and management. Some studies that provide an overview of the general protocols fail to examine the options based on the general community's socioeconomic status and the resources available in a particular area. The education level of physicians and technicians as well as the facilities/technologies available for those suffering from pelvic floor conditions is generally lacking in regions with an overall low socioeconomic status.

This paper aims to examine artificial intelligence as a means of cost-effectively diagnosing patients by detecting pressure changes within regions of interest (bladder and urethra), and as an avenue for suggesting low-cost treatments for women that suffer from urinary incontinence in low-resource communities such as South Asia and Africa.

\section{Literature Review}

\subsection{Methods}

A literature search was performed using a variety of databases including, but not limited to, the university's library database which is linked to Springer, PubMed, Wiley InterScience, and others. The authors included books, review articles, tri- 
al-based studies, case studies, and proposal reports to examine potential tools for the utilization of artificial intelligence to assist in diagnosing and managing urinary incontinence. The various resources were synthesized and initially screened based on their titles and abstracts. When the title of a paper or its abstract matched the relevant keywords of: "urinary incontinence", "artificial intelligence and urinary incontinence", "OGBYN and AI in low-resource locations", "AI and pelvic floor injury complications in [low-resource location name]", the resource was saved for further analysis and review.

\subsection{General Overview of Diagnosis and Management of UI}

Disturbances in the lower urinary tract cause urinary incontinence-typically due to dysfunction of the urethral sphincter, bladder, and pelvic floor structures. Vaginal delivery, hysterectomy, spinal cord injury, and/or menopause are also associated with UI. According to a study performed by Bai (2002), about $63 \%$ of cases involving damage to the pelvic floor, causing pelvic organ prolapse, would be then accompanied by stress UI [6]. Once there is damage originating from childbirth, which would result in loss of pelvic floor function, further deterioration of the urethral sphincter's muscle would cause the detrusor's overactivity [7]. Bladder dysfunction due to spinal cord injury (called a neurogenic bladder) either by childbirth or other means would allow a loss of the neural control of urination due to the bladder's uncoordinated contractions and the overactivity of the detrusor [8]. Hysterectomy is another avenue where the pelvic floor function may be altered. In Heydari's (2017) study, those who underwent hysterectomy found that they had a 6.3 times higher risk of developing severe incontinence than those that did not undergo the procedure [9]. Besides trauma and surgeries, one of the most significant factors for (mixed) urinary incontinence is age-where between $3 \%$ to $17 \%$ of women had a moderate to severe prevalence of UI with a higher prevalence amongst older-aged women (70+) [10]. Bear in mind that urinary incontinence is not a typical result of aging.

Patients who are suspected of having urinary incontinence would present to a physician. Physicians would follow a standardized approach to diagnose the condition and treat the disease. Khandelwal and Kistler (2013) designed a standardized system to examine UI. They utilized methods that included "consensus, disease-oriented evidence, done in practice, expert opinion, or evaluated in series of cases". They used three incontinence questions about leakage of urine, the cough stress test for stress UI, the PVR (postvoid residual urine) measurement, and a voiding diary [11]. Figure 1 represents the standardized procedure to diagnose UI, adapted from Khandelwal and Kistler (2013).

The Agency for Health Research and Quality (AHRQ) developed a systemic review in 2010, discussing various management protocols for urinary incontinence [12]. Table 1 lists three approaches of intervention for urinary incontinence adapted from AHRQ and other sources. 


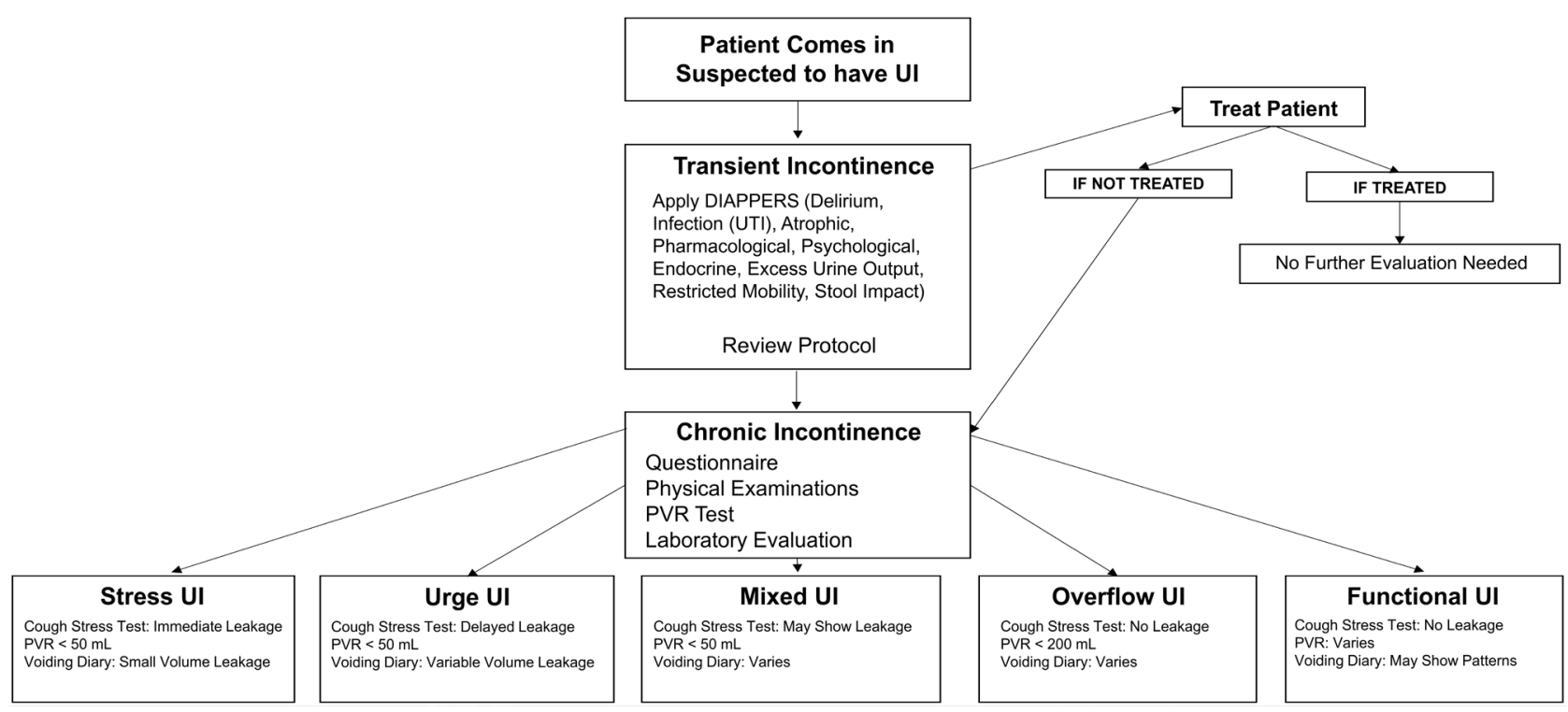

Figure 1. A standardized approach for diagnosing urinary incontinence. Patient would come into the clinic and is suspected to have UI. Assume that the patient is going through a transient phase of the condition-apply the DIAPPERS protocol and work on treating the patient. If the patient is not treated, the condition can advance to a chronic state, which would require further evaluation of the type of condition the patient is suffering from. Clinical management will then follow upon a diagnosis (adapted from Khandelwal and Kistler (2013)).

Table 1. Methods of intervention for urinary incontinence.

Exercise Based Approach Kegels pelvic floor exercise [12] [13]

Seratonin-noradrenaline uptake inhibitors-duloxetine [12]

Estrogens [12]

Allopathic Medicine Antimuscarinic drugs-trospium, darifenacin [12]

Mixed Action drugs-flavoxate, oxybutynin [12]

Medicinal Approach

Toxins-botulinum toxin, resiniferatoxin [12]

Gosha-jinki-gan [14]

Complimentary/Natural Medicine Capsaicin (exclude seeds) [14]

Pumpkin seed oil [15]

Vaginal cones-weighted device and is held by contraction of pelvic floor muscles to keep the device in place [12]

Device-Based Approach Pessaries-inserted into the vagina to maintain the location of organs in the pelvic region [12]

Low voltage electrical stimulation-stimulates muscles or nerves to reduce incontinence [12]

\subsection{Artificial Intelligence}

\subsubsection{General Overview}

Artificial Intelligence (AI) has been widely regarded as the future of the twenty-first century. AI is used in every primary industry, including the healthcare industry, to advance health care and improve patient outcomes. Literature has often debated the definition of AI as there is a lack of clarity around what AI entails. Today we refer to the umbrella term of AI as "machines that respond to 
stimulation consistent with traditional responses from humans, given the human capacity for contemplation, judgment, and intention" [16]. There are six main subsets of artificial intelligence, as described in Table 2 [17].

\subsubsection{General Applications in Healthcare}

Artificial intelligence has become a revolutionary intelligence system in the healthcare industry in recent years-with improvements being made in diagnosis, patient monitoring, and precision-based medicine. Table 3 describes certain AI-based healthcare technologies that utilize specific subsystems of AI-as mentioned by Wahl (2018) and Davenport (2019) [18] [19].

A study conducted in 2016 revealed that physicians spend $27 \%$ of their office days on direct patient interaction and spent nearly $50 \%$ of their days keeping up with electronic hospital records (EHR) and clerical desk work [20]. AI has been implemented to help primary care physicians in this scenario by helping take notes, analyze discussions with patients and enter required information directly into EHR systems, which has reduced manual labor for physicians, and as an effect, increased meaningful patient-physician interactions [21]. AI is rapidly growing, and investment in AI technology for the medical industry is expected to grow from $\$ 2.1$ billion to $\$ 36.1$ billion by 2025 as more advancements are made in this field [22]. AI will expand in the healthcare industry to support physicians, increase accuracy, and reduce medical errors in various ways.

Table 2. Six main subsets of artificial intelligence.

\begin{tabular}{cc}
\hline Machine Learning (ML) & $\begin{array}{r}\text { Complex math logic to decipher data based on patterns } \\
\text { Neural Networks }\end{array}$ \\
Robotics & $\begin{array}{r}\text { Replication of the human brain as an infinite number of neurons } \\
\text { at the machine level (works in conjunction with ML) } \\
\text { Conduction of tasks to improve efficiency (computer } \\
\text { systems deal with their control and intelligence) }\end{array}$ \\
Expert Systems & $\begin{array}{r}\text { computerized system that mimics the decision-making } \\
\text { of a human via reasoning (if-then rules) } \\
\text { Reasoning technique against uncertainties and } \\
\text { inaccuracies of a condition }\end{array}$ \\
Natural Language Processing & Processing of the human language by computerized systems \\
\hline
\end{tabular}

Table 3. Healthcare technologies involving the subsystems of artificial intelligence.

\begin{tabular}{cc}
\hline Machine Learning (ML) & $\begin{array}{c}\text { Precision-based medicine-using large datasets and applying } \\
\text { logic for diagnosis, therapeutic intervention, and prognosis }\end{array}$ \\
Neural Networks & $\begin{array}{r}\text { Categorization of diseases-using Artificial Neural Networks } \\
\text { (ANN) and Convolutional Neural Networks (CNN) }\end{array}$ \\
Deep Learning Models & Diagnosis (early and advanced) \\
Natural Language Processing & Documentation and conversation \\
Robotics & Advance surgical robots
\end{tabular}




\subsubsection{General Applications in Women's Health}

In women's health, including obstetrics and gynecology (OBGYN), the use of artificial intelligence has allowed improvements in protocol efficiency and delivery. Examples of general applications of AI in women's health include fetal heart monitoring, gestational diabetes mellitus, and in vitro fertilization. A summary of these applications is given in Table 4.

\subsection{Utilization of AI in Low Resourced Locations for UI Diagnosis and Management}

Developing nations and communities with low socioeconomic statuses such as Pakistan, India, and sub-Saharan Africa have suffered from the high burden of diseases, lack of trained healthcare providers and healthcare delivery systems [31]. As urinary incontinence is an underrepresented and undertreated disease, it is even more so in the regions with low socioeconomic backgrounds due to cultural reasons (neglect towards women) and the lack of adequate healthcare delivery [32]. However, using artificial intelligent systems, we could utilize these systems to improve efficiency in diagnosis, documentation, and patient conversation and increase the provided healthcare quality. This would ease the burden from the limited number of physicians by automating tedious workflows that can train over time, given new data sets, and provide more robust solutions [33].

\subsubsection{Current UI Diagnostic Modalities to Use with AI Systems}

In diagnosis and management, populations in low socioeconomic backgrounds do not have the means to afford treatment or have an unawareness of why they are suffering from dysfunction in the bladder and pelvic regions that cause urinary incontinence. Enabling artificial intelligence to diagnose patients with UI would improve healthcare access and ensure the quality of diagnoses for the underrepresented populations. The AI system would rely on current UI diagnostic modalities for its input system.

In Garcia del Salto's (2014) study, they noted that pelvic floor prolapses were frequently misidentified and proposed to use magnetic resonance imaging (MRI) that would visualize the pelvic floor's compartments to achieve more accurate diagnoses [34]. In Wei et al. (2017), they argued that although MRI would deliver high-quality images and data for patient-specific 3D reconstruction of the female pelvic floor for diagnoses, its utilization may be restrained due to the high expense and limited availability [35]. Ultrasound (US) imaging however is a low-cost modality that can be used to help build 3D models of the pelvic floor. Wei et al. (2017) proposed to utilize the high-resolution endo-vaginal ultrasonography to create accurate pelvic floor models to evaluate the pelvic floor dysfunctions and reduce injuries [35]. Emerging technologies utilizing ultrasound were highlighted by Shobeiri and Manonai (2014) including transperineal ultrasound (TPUS) to view the pelvic floor region with anatomical access, and the endocavitary ultrasound and real-time elastography (RTE) that uses the differences in the radiofrequency signals and differences of elastic properties in tissues 
to yield additional information about anatomical structures to enhance the information of the image [36]. Other emerging techniques were mentioned by Kim, Egorov, and Shobeiri (2017) including using Ultrasound Elasticity Imaging to provide visual information of the elasticity and anatomical structure, Acoustic Radiation Force Impulse (ARFI) and Ultrasonic Shear Wave Elasticity Imaging (SWEI) that are operator-independent and provide images of relative difference in tissue difference, and Functional Tactile Imaging (FTI) which would translate muscular activity for dynamic pressure patterns with respects to time [37]. An example of a device using FTI is the Vaginal Tactile Imaging (VTI) probe which acquires pressure response from the vaginal wall [37]. Instances, where the FTI modality including the VTI probe can be used, is to study the voluntary and involuntary reflex contractions, involuntary relaxation, or a Valsalva maneuver [37]. Table 5 compares the practicality of UI diagnosis methods to use with artificial intelligence in low-resource locations.

Table 4. AI applications for women's health.

\begin{tabular}{|c|c|c|}
\hline Application & Summary of Application & Relevant Studies using AI for Application \\
\hline $\begin{array}{l}\text { Fetal heart monitoring } \\
\text { (FHR) }\end{array}$ & $\begin{array}{l}\text { FHR is vital information when assessing the health } \\
\text { status of a fetus. Its use is a part of the standard } \\
\text { procedure for intrapartum assessment for fetal } \\
\text { well-being and aids in diagnosing cardiac disorders } \\
\text { [23]. }\end{array}$ & $\begin{array}{l}\text { Computer model CAFE (computer-aided fetal evaluator)- } \\
\text { a hybrid system that recorded the FHR and uterine contrac- } \\
\text { tion signals and proceeded to make decisions as an expert } \\
\text { using artificial neural networks. } \\
\text { The system could also detect sudden changes in the baseline, } \\
\text { highlighting anomalies to physicians [24]. } \\
\text { Computer-Aided Diagnosis (CAD) systems-uses CNNs } \\
\text { that process signals from a database and self-learn essential } \\
\text { features from the FHR signal for fetal asphyxia. } \\
\text { This system's self-learning ability allowed for a higher } \\
\text { accuracy rate as the network was able to automatically } \\
\text { extract features from the signal, which allowed for less } \\
\text { loss of information [25]. }\end{array}$ \\
\hline $\begin{array}{l}\text { Gestational diabetes } \\
\text { mellitus (GDM) }\end{array}$ & $\begin{array}{l}\text { GDM occurs when carbohydrate intolerance is } \\
\text { recognized for the first time during pregnancy. } \\
\text { Obesity, maternal age, and miscarriages are among } \\
\text { the risk factors associated with women who have } \\
\text { GDM. } \\
\text { For pregnant mothers, the United States Preventive } \\
\text { Services Taskforce recommends screening protocols } \\
\text { for GDM, which can often be overlooked for pregnant } \\
\text { women that live in low to middle-income areas or } \\
\text { countries due to the lack of availability [26]. }\end{array}$ & $\begin{array}{l}\text { Online Calculator Screening tool for GDM-uses patient } \\
\text { risk factors such as high blood pressure, smoking, weight, } \\
\text { diet, and ethnicity. Calculates risk for GDM using ANN and } \\
\text { backpropagation methods. Patients upload data from surveys, } \\
\text { and the calculator outputs the GDM risk factor. Polak and } \\
\text { Mendyk (2004) used this method on the record of } \\
2551 \text { women, of which } 91 \text { were diagnosed with GDM. } \\
\text { The calculator had an accuracy of 70\% where it detected } \\
\text { true positive GDM-but with more data and development } \\
\text { of the ANN, accuracy would increase and become more } \\
\text { economically efficient [27] [28]. }\end{array}$ \\
\hline
\end{tabular}


Table 5. Practicality of UI diagnosis modalities in combination with artificial-intelligentsystems in low-resource areas.

\begin{tabular}{|c|c|c|c|}
\hline Modality & Type & $\begin{array}{l}\text { Practical to use } \\
\text { alongside AI System } \\
\text { in Low Resourced } \\
\text { Location (Yes/No) }\end{array}$ & $\begin{array}{l}\text { How it can be used with AI System } \\
\text { in Low-Resource Location }\end{array}$ \\
\hline $\begin{array}{l}\text { Magnetic resonance } \\
\text { imaging }\end{array}$ & --- & No & Not usable-costly, limited availability \\
\hline \multirow{3}{*}{ Ultrasound } & TPUS & Yes & $\begin{array}{c}\text { Very simple to use diagnostic tool that } \\
\text { is used in multiple specialties to study } \\
\text { vaginal anatomy }\end{array}$ \\
\hline & RTE & Yes & $\begin{array}{l}\text { Widely used system since the early 1990s } \\
\text { (useful for the visual information as inputs to } \\
\text { an AI system for diagnosis of complication) }\end{array}$ \\
\hline & $\begin{array}{l}\text { ARFI and } \\
\text { SWEI }\end{array}$ & Yes & $\begin{array}{l}\text { Monitoring the radiation force region of } \\
\text { excitement and then generate the images } \\
\text { of the relative differences in tissue stiffness } \\
\text { in the cervix (SWEI) }\end{array}$ \\
\hline $\begin{array}{l}\text { Functional tactile } \\
\text { imaging }\end{array}$ & VTI & Yes & $\begin{array}{l}\text { Measure muscular activity into dynamic } \\
\text { pressure patterns for a particular location } \\
\text { (can be used for inputs to AI system for } \\
\text { diagnostic and/or treatment purposes) }\end{array}$ \\
\hline
\end{tabular}

Ultimately, after diagnosis, the physician would provide a treatment plan to the patients, which can be challenging considering the limited availability of resources in areas with low socioeconomic status. To assist the physicians to deliver specific treatment and management protocols (whether it be drugs, therapeutic devices, or surgery), precision or personalized medicine using AI systems can be used using the patient's data against large datasets to individualize care from the diagnosis to treatment selection and control, which would be optimized when new therapies and diagnostic methods come about [38].

\subsubsection{AI System Methods for UI}

For an AI System intended to be used on the diagnosis and management of UI, it needs to be able to parse the input data for it to accurately report diagnosis and potentially provide intervention and therapeutics to the patient. This means that the system needs to be sensitive and adaptable to different situations, such as in the case of patients residing in low socioeconomic regions where certain factors, either patient specific or availability of medical techniques and knowledge can influence the decision outputs of the system.

In a study by Burton (2019), three different machine learning algorithms (random forest, neural network, and extreme gradient boosting) were used to screen samples of urine for the detection of urinary tract infection (UTI) while factoring in demographics, historical urine culture results, and clinical details in 
which 212,554 reports were generated with classification sensitivity above $95 \%$ [39]. The study demonstrated that AI systems could reduce the diagnostic workload without compromising UTI detection; the same machine learning algorithms can be adapted to the detection of urinary incontinence. Barzegari's (2018) study investigated urethral pressure with particular parameters on patients with stress UI and ultimately found that if the trained artificial neural network (ANN) model were utilized, it would be an effective means to diagnose patients with stress UI [40]. In Geramipour's (2015) study with neural networks, real-time estimation of the bladder pressure using ANN was proposed and measurements using electroneurograms (ENG) on three rats, in which the neural network model can provide accurate assessment and prediction of bladder pressure and then stimulate it to increase bladder capacity to reduce incontinence [41]. In Luarikkala (1999), a system based on genetic algorithms, Galactica, was developed to devise accurate diagnostic rules for stress, mixed and urge UI. Being trained on 1105 datasets with testing performed on 485 datasets, the Galactica had a mean prediction accuracy of UI diagnosis of $90 \%$, with mean descriptive accuracy of UI diagnosis of $88 \%$ [42]. Galactica was compared against other models such as $k$-means cluster analysis, C.4.5 (rule-based and decision tree-based), and a random bit climber. The C.4.5 (rule-based) had the highest mean prediction and descriptive accuracy with $91 \%$ and $92 \%$, respectively. Galactica was compared with C.4.5 (rule-based) and was similar in their mean accuracies [42]. The Galactica system, although performed well, hasn't been used recently in clinical studies, which suggests that this system would not be of much use in an AI system being deployed in low-resource locations [42]. Lopes (2009) referenced three main categories of AI systems for diagnosis of UI including rule-based logic systems such as ALTURIN.EXP using EXPERTMD and ALTURIN.SDD (sensibility and specificity upper to $98 \%$ ), genetic algorithm-based systems such as Galactica (mentioned above from Laurikka, 1999), and fuzzy logic-based approved by NANDA (determine diagnosis in total accordance with panel for $79.5 \%$ of cases with $1.5 \%$ error) [43]. Hung (2019) utilized DeepSurv's deep learning model to predict UI after a robot-assisted radical prostatectomy (RARP) with performance metrics coming from 100 patients, with a mean absolute error of 85.9 and confidence interval of 0.6 [44]. Karam et al. (2016) developed a fast, adaptive, and accurate real-time method, called Context-Aware Thresholding (CAT) for classifying bladder events with pressure data acquired from 14 human subjects [45]. The CAT algorithm was tested against other algorithms for accuracy-Static Detrusor Thresholding (SDT), Global Detrusor Thresholding (GDT), Global Vesical Thresholding (GVT), Hybrid Detrusor Thresholding (HDT), and Adaptive Vesical Thresholding (AVT) [45]. In their results, the CAT algorithm displayed high accuracy of $97 \%$ for overactive bladder event classification with a $1.34 \%$ false positivity rate. AVT also displayed accurate results with $95 \%$ classification and a $1.39 \%$ false positivity rate. The other algorithms tested (SDT, HDT, GDT, and GVT) had an average of $74.25 \%$ accuracy with high false positivity rates of 
20.88\% (SDT) and 18.59\% (HDT) [45]. In Huang (2007), a computer-aided vector-based perineal ultrasound was used with an MLP Neural Network to diagnose urodynamic stress incontinence (USI) amongst 48 women with 36 of whom had USI. The model was trained with a backpropagation algorithm to minimize output distortion and then validated using the $k$-fold cross-validation for performance analyses [46]. The system attained accurate results with $91.7 \%$ accuracy of diagnosis USI with the computer-aided perineal ultrasound [46].

The studies above suggested that these systems can be trained over time, which means that the speed of the system could improve, the number of false negatives could decrease, and therefore an increased accuracy in diagnosis and determination of proper intervention methods [47]. Table 6 represents a summary of most of the AI systems mentioned above which may be useful to deploy to low-resource locations due to overall good performance results and the ability that these systems have to train over time.

Table 6. Summary of the AI methods for UI.

\begin{tabular}{|c|c|c|c|c|}
\hline Study & Clinical Purpose & AI Methods & $\begin{array}{c}\text { Number of } \\
\text { Datasets/Subjects }\end{array}$ & $\begin{array}{l}\text { Performance Results } \\
\text { of Model }\end{array}$ \\
\hline Burton, 2019 & $\begin{array}{l}\text { Diagnosis of urinary } \\
\text { tract infection }\end{array}$ & $\begin{array}{l}\text { Random forest; neural net- } \\
\text { work; extreme } \\
\text { gradient boosting }\end{array}$ & 212,554 reports & $\begin{array}{c}\text { Classification sensitivity } \\
\text { above } 95 \%\end{array}$ \\
\hline $\begin{array}{l}\text { Barzegari, } \\
2018\end{array}$ & Diagnosis of stress UI & MLP ANN & $\begin{array}{l}6453 \text { data points } \\
\text { (22 patients) }\end{array}$ & N/A \\
\hline $\begin{array}{l}\text { Geramipour, } \\
2015\end{array}$ & $\begin{array}{l}\text { Real-time estimation of } \\
\text { bladder pressure and } \\
\text { stimulation to } \\
\text { reduce incontinence }\end{array}$ & $\begin{array}{l}\text { MLP feed forward network } \\
\text { levenberg-marquardt } \\
\text { (for training) }\end{array}$ & 3 rats & $\begin{array}{l}\text { Tracking performance at } 8.89 \% \\
\text { (neural network model); } \\
\text { 5-s bladder prediction } \\
\text { error is about } 10.54 \%\end{array}$ \\
\hline $\begin{array}{c}\text { Laurikkala, } \\
1999\end{array}$ & $\begin{array}{l}\text { Diagnose female urinary } \\
\text { incontinence }\end{array}$ & $\begin{array}{l}\text { Genetic algorithm-based } \\
\text { galactica; C. } 4.5\end{array}$ & $\begin{array}{l}\text { Trained on } 1105 \text { sets; } \\
\text { tested on } 485\end{array}$ & $\begin{array}{l}\text { Mean prediction accuracy of } \\
91 \% \text { (C.4.5 rule-based) } \\
\text { and } 90 \% \text { (Galactica); } \\
\text { Mean descriptive accuracy } \\
\text { of } 92 \% \text { (C.4.5 rule-based) } \\
\text { and } 88 \% \text { (Galactica) }\end{array}$ \\
\hline $\begin{array}{l}\text { Hung et al., } \\
2019\end{array}$ & $\begin{array}{c}\text { Prediction of urinary } \\
\text { continence after robot-assisted } \\
\text { radical prostatectomy (RARP) }\end{array}$ & $\begin{array}{l}\text { Deep learning model } \\
\text { (DeepSurv) }\end{array}$ & $\begin{array}{l}\text { Automated performance } \\
\text { metrics acquired from } \\
100 \text { patients }\end{array}$ & $\begin{array}{c}\text { Achieved the lowest mean } \\
\text { absolute error (MAE) of 85.9; } \\
\text { CI of } 0.6\end{array}$ \\
\hline $\begin{array}{l}\text { Karem et al., } \\
\quad 2016\end{array}$ & $\begin{array}{l}\text { Classification of bladder } \\
\text { events such as detrusor } \\
\text { contraction for urinary } \\
\text { incontinence diagnosis }\end{array}$ & $\begin{array}{c}\text { Context-Aware } \\
\text { Thresholding (CAT) } \\
\text { algorithm }\end{array}$ & $\begin{array}{l}14 \text { human subjects; } \\
\text { vesical and abdominal } \\
\text { pressure data from } 64 \text { tracings } \\
\text { from the human subjects }\end{array}$ & $\begin{array}{c}97 \% \text { accuracy (CAT); } \\
1.34 \text { false positivity rate (CAT) }\end{array}$ \\
\hline Huang, 2007 & $\begin{array}{c}\text { Diagnosis of urodynamic } \\
\text { Stress incontinence (USI) } \\
\text { with computer-aided } \\
\text { perineal ultrasound }\end{array}$ & $\begin{array}{l}\text { MLP neural network } \\
\text { with backpropagation } \\
\text { algorithm for training }\end{array}$ & $\begin{array}{l}48 \text { women }-36 \text { with USI; } \\
12 \text { normal }\end{array}$ & $\begin{array}{c}\text { 91.7\% accuracy; } \\
\text { 94.4\% sensitivity; } \\
94.4 \% \text { positive predictive value; } \\
83.3 \% \text { negative predictive value }\end{array}$ \\
\hline
\end{tabular}




\section{Discussion}

Artificial intelligence and machine learning have proven to help support physicians and deliver efficient methods for diagnosis. AI can diagnose patients with high accuracy and provide personalized treatment protocols for managing the disease. AI systems are Artificial Intelligence and can become critical in diagnosing and managing urinary incontinence worldwide due to it being an undertreated and underrepresented complication. These systems would also likely ease the burden on the already lacking healthcare systems in certain parts of the world. Based on the reviewed studies, an expert AI system can be developed as a cost-efficient invasive or non-invasive tool that would utilize ANN or CNN architecture and other sensors to measure, detect, and deliver therapeutic (i.e., electric stimulation) to control the bladder and pelvic floor muscles. The system should have the capability to monitor the patient's health over time and recommend and deliver treatment and management options, and low-cost medical devices, such as pessaries-depending on the capabilities the system has and the resources available in the area [48].

The patient's experience could be enhanced with neuromodulation devices that have ML capabilities on-board and can access the databases, and provide stimulation to sacral nerves or tibia if there is an early detection of urinal incontinence (based on contractions, urethral pressure, etc.) so that bladder could control the urine flow-and these can be achieved via Percutaneous Tibial Neuromodulation (PTNM) or Percutaneous Tibial Neurostimulation (PTNS), which are non-implantable solutions or sacral neuromodulation (SNM) which is an implantable solution [49]. The devices that have PTNM, PTNS, and SNM do exist; however, they currently do not have a robust ML algorithm capability onboard that can be designed specifically for each patient. To improve patient outcomes, one could create a low-cost system that contains diagnostic neurostimulation abilities that would be able to learn in an unsupervised environment. Typical systems would have patients use a mobile network to turn on an implanted device. Still, a pessary-like device can be developed that would detect and stimulate with variable current based on pressure.

Figure 2 represents a proposed model, inspired by Barzegari (2018) that could be implemented in low-cost medical devices tailored to patients in low socioeconomic regions that would take in five input values that would go through a multilayer perceptron (MLP) feed forward artificial neural network (ANN) model to get four to five different outputs.

The five inputs that will be fed into the system are the position/location of the pelvic floor and urethra, the age and demographics of the patient, the initial 2D scan and 3D scan of the patient's pelvic floor region. The data will go through a multilayer hidden layer where the complex computation and logic rules are applied to the input parameters. This would include the system going through medical databases full of the data related to the pelvic floor prolapse and urinary incontinence based on the input layer. The output would be the urethral 


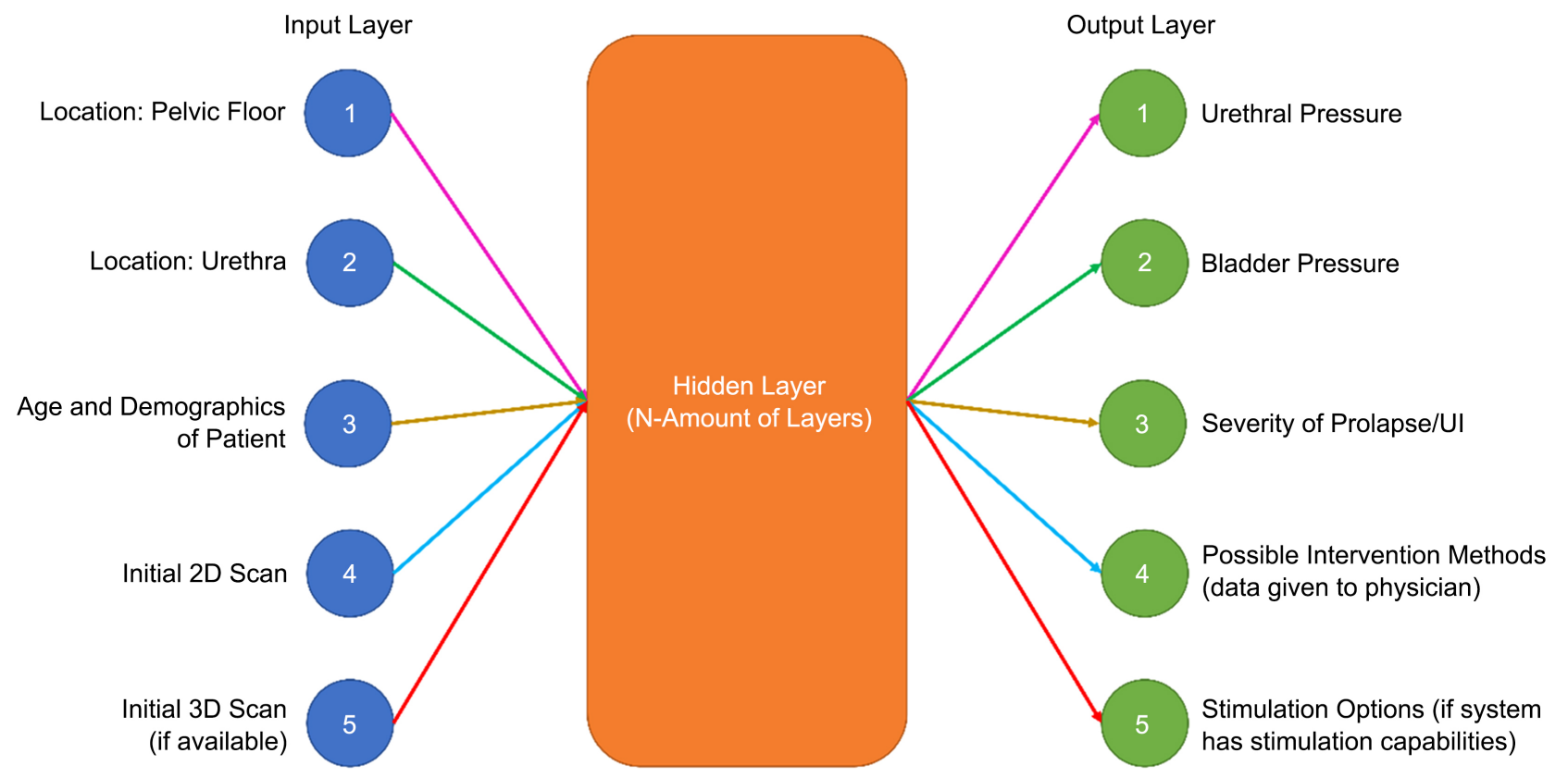

Figure 2. A Multilayer Perceptron (MLP) Feedforward Artificial Neural Network (ANN) Model proposed to use in devices containing diagnostic and neuromodulation/neurostimulation capabilities. The proposed model will input four to five inputs based on position, patient demographics and ultrasound-based scans. The multilayer hidden layer is the layer where complex computation and logic rules are applied to the input layer parameters to obtain the output values of the pressure, severity of complication and intervention options (where the patient is able to accept or deny treatment).

and bladder pressure, the severity of the and urinary incontinence, possible intervention methods where data is given to the physician and relayed to the patient, and the possible stimulation options. The stimulation modality would be possible if the device utilizing the proposed MLP model has the capabilities of administering the stimulation or other intervention methods. The model in Barzegari's (2018) research trained the neural network based on a fairly small dataset of 22 patients and had a good relationship between the model outputs and medical analysis. If the proposed model in Figure 2 can be utilized with a larger group of patients, indicating a larger dataset, then it would be possible to test the accuracy of calculated pressures, disease severity, and the ability to administer intervention mechanisms. Once the accuracy of above $90 \%$ is reached of the system, with a low false positivity rate, it can be utilized effectively in regions like in South Asia and Africa in tackling the urinary incontinence problem-the system could potentially diagnose more patients, limit the burden on the physicians and the healthcare system, and provide low-cost (or even free) treatment for patients.

\section{Further Implications}

Despite the AI model proposed in Figure 2 having the likelihood of being accurate, the implementations of the AI model or the AI system into healthcare practices in the developing regions will be challenging. The first main challenge is utilizing patient data in those specific regions and training the data based on 
medical databases to come up with an outcome, as some techniques that are used in a developed region may not apply for a low-resource location. Factors such as compliance of the local healthcare system and their respective government regulations will have to be considered in addition to the patient preference (accepting or denying treatment) and cost to maintain the system (either cost to patient or cost to the client such as government or healthcare operator). In certain countries, health insurance coverage (or the lack of it) will be an important consideration with regard to implementation. Finally, an overall acceptance of advance technologies being utilized to solve medical issues will be needed, as in certain regions (villages and tribes, for instance), they may not have positive regard for technologies. Educating the patients will be necessary in order to have a smooth transition into utilizing personalized care systems for the patients suffering from urinary incontinence with low socioeconomic backgrounds.

\section{Acknowledgements}

We would like to thank the Department of Bioengineering at George Mason University for supporting the publication expenses.

\section{Conflicts of Interest}

The authors declare no conflicts of interest regarding the publication of this paper.

\section{References}

[1] Global Forum on Incontinence (2018) About Incontinence. https://www.gfiforum.com/incontinence

[2] Aoki, Y., Brown, H.W., Brubaker, L., Cornu, J.N., Daly, J.O. and Cartwright, R. (2017) Urinary Incontinence in Women. Nature Reviews Disease Primers, 3, Article ID: 17042 .

[3] Haylen, B.T., de Ridder, D., Freeman, R.M., Swift, S.E., Berghmans, B., Lee, J., et al. (2010) An International Urogynecological Association (IUGA)/International Continence Society (ICS) Joint Report on the Terminology for Female Pelvic Floor Dysfunction. International Urogynecology Journal, 21, 5-26. https://doi.org/10.1007/s00192-009-0976-9

[4] Steers, W.D. (2002) Pathophysiology of Overactive Bladder and Urge Urinary Incontinence. Reviews in Urology, 4, S7-S18.

[5] Biswas, B., Bhattacharyya, A., Dasgupta, A., Karmakar, A., Mallick, N. and Sembiah, S. (2017) Urinary Incontinence, Its Risk Factors, and Quality of Life: A Study among Women Aged 50 Years and above in a Rural Health Facility of West Bengal. Journal of Mid-Life Health, 8, 130-136. https://doi.org/10.4103/jmh.JMH_62_17

[6] Bai, S.W., Jeon, M.J., Kim, J.Y., Chung, K.A., Kim, S.K. and Park, K.H. (2002) Relationship between Stress Urinary Incontinence and Pelvic Organ Prolapsed. International Urogynecology Journal, 13, 256-260. https://doi.org/10.1007/s001920200053

[7] Norton, P. and Brubaker, L. (2006) Urinary Incontinence in Women. The Lancet, 367, 57-56. https://doi.org/10.1016/S0140-6736(06)67925-7

[8] Taweel, W.A. and Seyam, R. (2015) Neurogenic Bladder in Spinal Cord Injury Pa- 
tients. Research and Reports in Urology, 7, 85-99.

https://doi.org/10.2147/RRU.S29644

[9] Heydari, F., Motaghed, Z. and Abbaszadeh, S. (2017) Relationship between Hysterectomy and Severity of Female Stress Urinary Incontinence. Electron Physician, 9 , 4678-4682. https://doi.org/10.19082/4678

[10] Nitti, V.W. (2001) The Prevalence of Urinary Incontinence. Reviews in Urology, 3, S2-S6.

[11] Khandelwal, C. and Kistler, C. (2013) Diagnosis of Urinary Incontinence. American Family Physician, 87, 543-550.

[12] Effective Health Care Program (2010) Diagnosis and Comparative Effectiveness of Treatments for Urinary Incontinence in Adult Women.

https://effectivehealthcare.ahrq.gov/products/urinary-incontinence-treatment/resea rch-protocol

[13] Gross, K.B. (2020) How to Strengthen Your Muscles to Eliminate Incontinence. https://www.iowaclinic.com/physical-therapy/how-to-strengthen-your-muscles-toeliminate-incontinence/

[14] Chughtai, B., Kavaler, E., Lee, R., Te, A., Kaplan, S.A. and Lowe, F. (2013) Use of Herbal Supplements for Overactive Bladder. Reviews in Urology, 15, 93-96.

[15] Nishimura, M., Ohkawara, T., Sato, H., Takeda, H. and Nishihira, J. (2014) Pumpkin Seed Oil Extracted from Cucurbita maxima Improves Urinary Disorder in $\mathrm{Hu}$ man Overactive Bladder. Journal of Traditional and Complementary Medicine, 4, 72-74. https://doi.org/10.4103/2225-4110.124355

[16] West, D.M. (2018) What Is Artificial Intelligence? Brookings. https://www.brookings.edu/research/what-is-artificial-intelligence/

[17] Tyagi, N. (2020) 6 Major Branches of Artificial Intelligence (AI). https://analyticssteps.com/blogs/6-major-branches-artificial-intelligence-ai

[18] Wahl, B., Cossy-Gantner, A., Germann, S. and Schwalbe, N.R. (2018) Artificial Intelligence (AI) and Global Health: How Can AI Contribute to Health in Resource-Poor Settings? BMJ Glob Health, 3, e000798.

https://doi.org/10.1136/bmjgh-2018-000798

[19] Davenport, T. and Kalakota, R. (2019) The Potential for Artificial Intelligence in Healthcare. Future Healthcare Journal, 6, 94-98. https://doi.org/10.7861/futurehosp.6-2-94

[20] Sinsky, C., Colligan, L., Li, L., Prgomet, M., Reynolds, S., Goeders, L., et al. (2016) Allocation of Physician Time in Ambulatory Practice: A Time and Motion Study in 4 Specialties. Annals of Internal Medicine, 165, 753-760. https://doi.org/10.7326/M16-0961

[21] Amisha, Malik, P., Pathania, M. and Rathaur, V.K. (2019) Overview of Artificial Intelligence in Medicine. Journal of Family Medicine and Primary Care, 8, 2328-2331. https://doi.org/10.4103/jfmpc.jfmpc_440_19

[22] Iftikhar, P., Kuijpers, M.V., Khayyat, A., Iftikhar, A. and Sa, M.D.D. (2020) Artificial Intelligence: A New Paradigm in Obstetrics and Gynecology Research and Clinical Practice. Cureus, 12, e7134. https://doi.org/10.7759/cureus.7124

[23] Blix, E., Maude, R., Hals, E., Kisa, S., Karlsen, E., Nohr, E.A., et al. (2019) Intermittent Auscultation Fetal Monitoring during Labour: A Systematic Scoping Review to Identify Methods, Effects, and Accuracy. PLOS ONE, 14, e0219573. https://doi.org/10.1371/journal.pone.0219573

[24] Guijarro-Berdiñas, B. and Alonso-Betanzos, A. (2002) Empirical Evaluation of a Hy- 
brid Intelligent Monitoring System Using Different Measures of Effectiveness. Artificial Intelligence in Medicine, 24, 71-96. https://doi.org/10.1016/S0933-3657(01)00091-4

[25] Zhao, Z., Deng, Y., Zhang, Y., Zhang, Y., Zhang, X. and Shao, L. (2019) DeepFHR: Intelligent Prediction of Fetal Acidemia Using Fetal Heart Rate Signals Based on Convolutional Neural Network. BMC Medical Informatics and Decision Making, 19, Article No. 286. https://doi.org/10.1186/s12911-019-1007-5

[26] United States Preventive Services Taskforce (2014) Final Recommendation Statement: Gestational Diabetes Mellitus, Screening.

https://www.uspreventiveservicestaskforce.org/uspstf/document/RecommendationS tatementFinal/gestational-diabetes-mellitus-screening

[27] Polak, S. and Mendyk, A. (2004) Artificial Intelligence Technology as a Tool for Initial GDM Screening. Expert Systems with Applications, 26, 455-460. https://doi.org/10.1016/j.eswa.2003.10.005

[28] Shen, J., Chen, J., Zheng, Z., Zheng, J., Liu, Z., Song, J., et al. (2020) An Innovative Artificial Intelligence-Based App for the Diagnosis of Gestational Diabetes Mellitus (GDM-AI): Development Study. Journal of Medical Internet Research, 22, e21573. https://doi.org/10.2196/21573

[29] Siristatidis, C., Pouliakis, A., Chrelias, C. and Kassanos, D. (2011) Artificial Intelligence in IVF: A Need. Systems Biology in Reproductive Medicine, 57, 179-185. https://doi.org/10.3109/19396368.2011.558607

[30] Guh, R.-S., Wu, T.-C.J. and Weng, S.-P. (2011) Integrating Genetic Algorithm and Decision Tree Learning for Assistance in Predicting in Vitro Fertilization Outcomes. Expert Systems with Applications, 38, 4437-4449. https://doi.org/10.1016/j.eswa.2010.09.112

[31] Orach, C.G. (2009) Health Equity: Challenges in Low Income Countries. African Health Sciences, 9, S49-S51.

[32] DW.COM. (2019) Access to Health Care a Distant Dream for Most Indian Women. https://www.dw.com/en/access-to-health-care-a-distant-dream-for-most-indian-wo men/a-50108512

[33] Hoodbhoy, Z., Hasan, B. and Siddiqui, K. (2019) Does Artificial Intelligence Have Any Role in Healthcare in Low Resource Settings? Journal of Medical Artificial Intelligence, 2, Article No. 13. https://doi.org/10.21037/jmai.2019.06.01

[34] García del Salto, L., de Miguel Criado, J., del Hoyo, L.F.A., Velasco, L.G., Rivas, P.F., Paradela, M.M., et al. (2014) MR Imaging-Based Assessment of the Female Pelvic Floor. RadioGraphics, 34, 1417-1439. https://doi.org/10.1148/rg.345140137

[35] Wei, Q., Sikdar, S., Chitnis, P., Rostaminia, G. and Abbas Shobeiri, S. (2017) Patient-Specific Studies of Pelvic Floor Biomechanics Using Imaging. In: Shobeiri, S.A., Ed., Practical Pelvic Floor Ultrasonography, Springer International Publishing, Cham, 337-344. https://doi.org/10.1007/978-3-319-52929-5_18

[36] Shobeiri, S.A. and Manonai, J.B. (2014) Emerging Imaging Technologies and Techniques. In: Shobeiri, S.A., Ed., Practical Pelvic Floor Ultrasonography, Springer International Publishing, New York, 195-210.

https://doi.org/10.1007/978-1-4614-8426-4_11

[37] Kim, K., Egorov, V. and Abbas Shobeiri, S. (2017) Emerging Imaging Technologies and Techniques. In: Shobeiri, S.A., Ed., Practical Pelvic Floor Ultrasonography, Springer International Publishing, Cham, 327-336. https://doi.org/10.1007/978-3-319-52929-5_17

[38] Ho, D., Quake, S.R., McCabe, E.R.B., Chng, W.J., Chow, E.K., Ding, X., et al. (2020) 
Enabling Technologies for Personalized and Precision Medicine. Trends in Biotechnology, 38, 497-518. https://doi.org/10.1016/j.tibtech.2019.12.021

[39] Burton, R.J., Albur, M., Eberl, M. and Cuff, S.M. (2019) Using Artificial Intelligence to Reduce Diagnostic Workload without Compromising Detection of Urinary Tract Infections. BMC Medical Informatics and Decision Making, 19, Article No. 171. https://doi.org/10.1186/s12911-019-0878-9

[40] Barzegari, M., Vahidi, B., Safarinejad, M.R. and Hashemipour, M. (2018) Pathological Analysis of Stress Urinary Incontinence in Females Using Artificial Neural Networks. http://arxiv.org/abs/1803.01843

[41] Geramipour, A., Makki, S. and Erfanian, A. (2015) Neural Network Based Forward Prediction of Bladder Pressure Using Pudendal Nerve Electrical Activity. 201537 th Annual International Conference of the IEEE Engineering in Medicine and Biology Society (EMBC), Milan, August 2015, 4745-4748.

https://doi.org/10.1109/EMBC.2015.7319454

[42] Laurikkala, J., Juhola, M., Lammi, S. and Viikki, K. (1999) Comparison of Genetic Algorithms and Other Classification Methods in the Diagnosis of Female Urinary Incontinence. Methods of Information in Medicine, 38, 125-131.

https://doi.org/10.1055/s-0038-1634175

[43] Lopes, M.H., Marin, H., Ortega, N.R.S. and Massad, E. (2009) The Use of Expert Systems on the Differential Diagnosis of Urinary Incontinence. Revista da Escola de Enfermagem da USP, 43, 704-710. https://doi.org/10.1590/S0080-62342009000300029

[44] Hung, A.J., Chen, J., Ghodoussipour, S., Oh, P.J., Liu, Z., Nguyen, J., et al. (2019) Deep Learning On automated Performance Metrics and Clinical Features to Predict Urinary Continence Recovery after Robot-Assisted Radical Prostatectomy. BJU International, 124, 487-495. https://doi.org/10.1111/bju.14735

[45] Karam, R., Bourbeau, D., Majerus, S., Makovey, I., Goldman, H.B., Damaser, M.S., et al. (2016) Real-Time Classification of Bladder Events for Effective Diagnosis and Treatment of Urinary Incontinence. IEEE Transactions on Biomedical Engineering, 63, 721-729. https://doi.org/10.1109/TBME.2015.2469604

[46] Huang, Y.-L. and Chen, H.-Y. (2007) Computer-Aided Diagnosis of Urodynamic Stress incontinence with Vector-Based Perineal Ultrasound Using Neural Networks. Ultrasound in Obstetrics \& Gynecology, 30, 1002-1006.

https://doi.org/10.1002/uog.4102

[47] Guo, J. and Li, B. (2018) The Application of Medical Artificial Intelligence Technology in Rural Areas of Developing Countries. Health Equity, 2, 174-181. https://doi.org/10.1089/heq.2018.0037

[48] Raney, J., Hong, C., Cioban, M., Yasuda, H. and Mo, C. (2020) Technology-Novel, Customizable Pessary for Pelvic Organ Prolapse Treatment. University of Pennsylvania-Penn Center for Innovation.

https://upenn.technologypublisher.com/technology/37651

[49] Medtronic (2020) Neuromodulation-Urinary and Bowel Incontinence. https://www.medtronic.com/us-en/healthcare-professionals/therapies-procedures/u rology/neuromodulation-therapies.html 